ORIGINAL ARTICLE

\title{
What is the patient really taking? Discrepancies between surgery and anesthesiology preoperative medication histories
}

\author{
S A Burda, D Hobson, P J Pronovost
}

Qual Saf Health Care 2005;14:414-416. doi: 10.1136/qshc.2005.014738

\begin{abstract}
See end of article for authors' affiliations

.....................

Correspondence to: S A Burda, The Johns Hopkins Hospital, Department of Pharmacy, 600 North Wolfe St, Carnegie 180, Baltimore, MD 21287-6180, USA; sburda1@jhmi.edu
\end{abstract}

Accepted for publication 15 August 2005
Background: Surgical patients may be at risk for medication discrepancies that may lead to medication errors because both the anesthesiologist and the surgeon write separate preoperative medication histories. Methods: A prospective observational study was conducted to examine the extent of medication and allergy discrepancies between surgical and anesthesia preoperative medication histories for patients admitted to two surgical intensive care units in an academic medical center.

Results: Of the 79 patient records reviewed, 58 (73\%) contained at least one discrepancy, 23\% had different allergy information, $56 \%$ had different preoperative medications, and $43 \%$ had different doses or dosing frequencies listed in the medication histories. Of the 988 allergies, medications, and doses or dosing frequencies documented in the two histories, $456(46 \%)$ contained discrepancies. Of these discrepancies, $20(5 \%)$ were due to different allergies, $293(64 \%)$ to different medications, and $143(31 \%)$ to different doses or dosing frequencies.

Conclusions: Discrepancies in preoperative medication histories between surgical and anesthesia records occur in most patients and further work is required to help improve agreement of patient medication histories between services.
$\mathrm{E}$ ach year a significant number of patients is harmed as a result of interaction with healthcare organizations. ${ }^{1}$ Medication errors are common and can lead to significant harm and expense. ${ }^{2-5}$ A major cause of medication errors has been attributed to prescribers' lack of essential drug and patient information at the time of ordering. ${ }^{67}$ As a result, many organizations are implementing interventions such as Computerized Provider Order Entry (CPOE) to reduce the rate of medication errors. ${ }^{89}$ Nevertheless, systems such as $\mathrm{CPOE}$ require that providers know which medications patients should be receiving.

Because both the anesthesiologist and the surgeon write separate preoperative medication histories, surgical patients may be at risk of having medication discrepancies. The purpose of this study was to determine the percentage of patients admitted postoperatively to an intensive care unit (ICU) who had a discrepancy between the anesthesiology and surgery preoperative medication histories. In addition, we examined the total number of allergy and medication regimen discrepancies between surgery and anesthesiology preoperative medication histories.

\section{METHODS}

The study was a prospective cohort study conducted in two surgical ICUs at an academic medical center. The WICU is a 14 bed ICU that cares for approximately 1200 surgical oncology patients per year, and the SICU is a 14 bed ICU that primarily cares for vascular, trauma, and transplant patients and admits approximately 1100 patients per year. In both ICUs care is provided by an intensivist led team that includes an attending intensivist, surgery and anesthesiology residents, nurse practitioners, pharmacists, and respiratory therapists.

The study population included all patients admitted postoperatively to the WICU or SICU from the operating room during April 2004. On admission, a pharmacist or ICU nurse reviewed the preoperative medication histories documented by the anesthesiology and surgery services. These preoperative medication histories were completed by a member of the surgery team either in a preoperative evaluation clinic or on admission to the hospital for surgery. The anesthesiologist completed a medication history during the preoperative evaluation before the patient went to the operating room.

\section{Outcome variables}

The primary outcome variable was the percentage of patients in whom a discrepancy in either allergy or medication regimen was found between the surgery and anesthesiology preoperative medication histories. Secondary outcome variables were the percentage of allergy and medication discrepancies between all medication regimens and allergy information documented in both medication histories. For both the primary and secondary outcomes, the discrepancies were further classified as different allergy, different medication, and different dose or dosing frequency.

Because we did not know the "true" medications or allergies, if a medication or allergy was listed on either the surgical or anesthesiology history we included it as a medication the patient should be taking or as a true allergy that should be documented in the medication history. To identify a discrepancy, we compared the allergy information and medication regimens documented in the surgery and anesthesiology preoperative medication histories at the time of ICU admission. Any differences between the two charts in allergies, medications, doses, or dose frequencies were considered discrepancies.

A standardized data collection tool was used by two investigators (SB, DH) who reviewed patient medication histories documented by both anesthesiology and surgery. For each patient, allergies, medications, doses, and dosing frequencies from both medication histories were compared. A discrepancy was documented if a difference in allergy, 
Table 1 Patients' records with discrepancies in medication regimens and allergy information in two ICUs

\begin{tabular}{llll}
\hline Discrepancy & $\begin{array}{l}\text { WICU } \\
(\mathbf{n = 3 4 )}\end{array}$ & $\begin{array}{l}\text { SICU } \\
(\mathbf{n = 4 5 )}\end{array}$ & $\begin{array}{l}\text { Total } \\
(\mathbf{n}=\mathbf{7 9})\end{array}$ \\
\hline $\begin{array}{l}\text { At least one discrepancy in allergy } \\
\text { or medication regimen }\end{array}$ & $27(79.0 \%)$ & $31(68.9 \%)$ & $58(73.4 \%)$ \\
Different allergy & $10(29.0 \%)^{*}$ & $8(18.0 \%)$ & $18(23.0 \%)$ \\
Different medication & $24(70.0 \%)^{*}$ & $20(44.0 \%)$ & $44(56.0 \%)$ \\
Different dose/dosing frequency & $13(38.0 \%)^{*}$ & $21(47.0 \%)$ & $34(43.0 \%)$ \\
\hline *Percentages will not add to 100\%. & & & \\
\end{tabular}

medication, dose, or dosing frequency was found between the two medication histories.

\section{Analysis of data}

The analysis was descriptive. The patient was the unit of analysis for our primary outcome variable (the percentage of patients for whom one or more discrepancies in their allergy information or their medication regimens were found between the anesthesiology or surgery preoperative medication histories), and the medication regimen or allergy was the unit of analysis for our secondary outcome variable (the percentage of medications and allergies for which a discrepancy was found). We further classified the discrepancy as a different allergy, different medication, and/or different dose or dosing frequency.

\section{RESULTS}

During the study period 34 patients were admitted to the WICU and 45 to the SICU; data were collected on all of these. Of the 79 patient records, discrepancies in allergy information or medication regimen were found in $58(73 \%)$. Of these, 18 $(23 \%)$ were due to different allergy, $44(56 \%)$ to different medication, and $34(43 \%)$ to different dose or dosing frequency (table 1). The rates were similar between the two ICUs.

Of the 988 medications, doses or dosing frequencies, and allergies reviewed in the study, $352(36 \%)$ contained a discrepancy. Of these discrepancies, $20(5 \%)$ were due to different allergy, $293(64 \%)$ to different medications, and 143 $(31 \%)$ to different dose or dosing frequency. Furthermore, we found that $20 \%$ of all allergies were different, $57 \%$ of all medications were different, and 39\% of all doses and dosing frequencies were different between the two medication histories. The rates were similar between the two ICUs (table 2).

\section{DISCUSSION}

Discrepancies between surgery and anesthesiology preoperative medication histories existed for most patients admitted postoperatively to our surgical ICUs. This poses a significant risk to patient safety and should be a focus of improvement efforts.

Agreement of patients' medication profiles is important to ensure that patients receive the correct medications and to reduce complications. While systems such as CPOE have helped to decrease medication errors, medical teams must first determine and agree upon what allergies and medications are appropriate for each patient. An ideal patient course demands not only an improved system approach, but also is dependent on reliable, consistent, and efficient communication among teams who care for a patient.

This study highlights the importance of communication among surgery and anesthesiology services in clarifying medications and allergies. One potential intervention to mitigate this issue is the use of medication reconciliation. We have shown that medication errors can be significantly reduced if ICU nurses review patients' medication orders before discharge from the ICU to identify and mitigate discrepancies between the medications received in the ICU and those on the transfer orders. Such a system, if applied in the preoperative evaluation center before surgery, may significantly improve medication safety. ${ }^{10}$ An alternative-or perhaps complementary-strategy would be to use a pharmacist in the preoperative area to reconcile differences in medication histories. ${ }^{11-14}$ Considering the magnitude of this problem, healthcare organizations should seek remedies.

This study has several limitations. Firstly, we conducted the study in an academic medical center and in patients admitted to an ICU. We may therefore have selected patients who are more ill and on more medications, and cannot comment on the magnitude of the problem in surgical patients not admitted to the ICU or at other hospitals. Nevertheless, the same caregivers take medication histories for ICU and non-ICU patients, raising the concern that this is also a problem in non-ICU patients. Secondly, we studied a relatively small number of patients; however, given that most patients $(73 \%)$ had a discrepancy and the rates were similar between the two ICUs, it is unlikely that a large sample size would have significantly changed our results. Thirdly, our method of defining medication or allergy discrepancies may be questionable. We strove for a practical approach that would be meaningful to caregivers. Finally, we studied patients with a high severity of illness who were being admitted to an ICU. Patients being admitted to regular hospital wards may be on less medication and thus have less risk for discrepancies. ${ }^{15}$

In conclusion, discrepancies exist between surgery and anesthesiology preoperative medication histories for most postoperative patients admitted to our surgical ICU. Efforts to resolve these discrepancies should be a high priority.

Table 2 Medication regimen and allergy discrepancies

\begin{tabular}{llll}
\hline Discrepancy & WICU & SICU & Total \\
\hline Allergy discrepancies & $11 / 36(30.5 \%)$ & $9 / 65(13.8 \%)$ & $20 / 101(19.8 \%)$ \\
Total medication regimen discrepancies & $120 / 245(49.0 \%)$ & $316 / 642(49.2 \%)$ & $436 / 887(49.1 \%)$ \\
Different dose/dosing frequency & $39 / 82(47.6 \%)$ & $104 / 286(36.3 \%)$ & $143 / 368(38.9 \%)$ \\
Different medication & $81 / 163(49.7 \%)$ & $212 / 356(59.5 \%)$ & $293 / 519(56.5 \%)$ \\
Overall discrepancies & $131 / 281(46.6 \%)$ & $325 / 702(46.3 \%)$ & $456 / 988(46.2 \%)$ \\
\hline
\end{tabular}




\section{Authors' affiliations}

S A Burda, Department of Pharmacy, Johns Hopkins Medical Institutions, Baltimore, MD, USA

D Hobson, Department of Surgical Nursing, Johns Hopkins Medical Institutions, Baltimore, MD, USA

P J Pronovost, Departments of Anesthesiology and Critical Care Medicine, Surgery, Health Policy and Management, Nursing; and the Center for Innovations in Quality Patient Care, Johns Hopkins Medical Institutions, Baltimore, MD, USA

This project was internally funded.

\section{REFERENCES}

1 Kohn L, Corrigan J, Donaldson M. To err is human: building a safer health system. Institute of Medicine Report. Washington, DC: National Academy Press, 1999.

2 Bates D, Spell N, Cullen D, et al. The costs of adverse drug events in hospitalized patients. JAMA 1997:277:307-11.

3 Johnson JA, Bootman U. Drug-related morbidity and mortality: a cost-ofillness model. Arch Intern Med 1995;155:1949-56.

4 Dean B, Schachter M, Vincent C, et al. Causes of prescribing errors in hospital inpatients: a prospective study. Lancet 2002;359:1373-8.
5 Lesar TS, Briceland LL, Delcoure $\mathrm{K}$, et al. Medication prescribing errors in a teaching hospital. JAMA 1990;17:2329-34.

6 Lesar TS, Briceland L, Stein DS. Factors related to errors in medication prescribing. JAMA 1997:4:312-7.

7 Leape LL, Bates DW, Cullen DJ, et al. Systems analysis of adverse drug events. JAMA 1995;274:35-43.

8 Bates DW, Cullen DJ, Laird N, et al. Incidence of adverse drug events and potential adverse drug events. JAMA 1995;274:29-34,

9 Bates DW, Leape LL, Cullen DJ, et al. Effect of computerized physician order entry and a team intervention on prevention of serious medication errors. JAMA 1998;280:1311-6

10 Pronovost $\mathrm{P}$, Weast $B$, Schwarz $M$, et al. Medication reconciliation: a practical tool to reduce the risk of medication errors. J Crit Care 2003; 18:201-5.

11 Bond CA, Raehl CL, Franke T. Clinical pharmacy services and hospital mortality rates. Pharmacotherapy 1999; 19:556-64.

12 Gurwich EL. Comparison of medication histories acquired by pharmacists and physicians. Am J Hosp Pharm 1983;40:1541-2.

13 Covington TR, Pfeiffer FG. The pharmacist-acquired medication history Am J Hosp Pharm 1972;29:692-5.

14 Wilson RS, Kabat HF. Pharmacist initiated patient drug histories. Am J Hosp Pharm 1971;28:49-53.

15 Cullen DJ, Sweitzer BJ, Bates DW, et al. Preventable adverse drug events in hospitalized patients: a comparative study of intensive care and general care units. Crit Care Med 1997;25:1289-97.

\section{$\mathrm{ECHO}$}

\section{UK initiative improves outcome for heart disease}

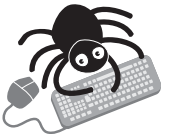

Please visit the Quality and Safety in Health Care website [www. qshc.com] for a link to the full text of this article.
$T$ wo years on, the National Service Framework (NSF) for coronary heart disease (CHD) in the UK has improved health outcomes, with lower mortality and more uniform and timely treatment, a retrospective before and after review in two London hospitals has shown. Setting clear, unambiguous targets within regularly audited guidelines can benefit care, say the study's authors.

Significant improvements directly related to the intervention in patients with acute coronary syndromes or myocardial infarction were lower mortality-falling by a third after the NSF was implemented in April 2000-and fewer patients with Q wave myocardial infarction and left ventricular failure; also more eligible patients received thrombolysis and sooner. However, increased use of ACE inhibitors and more referrals for revascularisation might be attributable to other guidelines, and use of $\beta$ blockers and statins increased in line with existing trends. The two patient groups were similar demographically, and the NSF was the only variable to account for the findings.

The review compared patients in the coronary care units of the east end London hospitals which serve 700000 people and where a database, set up in 1998, charts treatment and outcomes of all admissions with acute coronary diagnoses. During 1998-2002, 1993 patients in the 27 months before the NSF was implemented were compared with 1378 patients 21 months after.

The NSF was introduced by the government in March 2000 to curb deaths from CHD by $40 \%$ over 10 years and to end regional variation in treatment—or "postcode prescribing."

A Graham JJ, et al. Heart. Published Online First: 20 May 2005. doi: 10.1136/hrt.2004.051466 